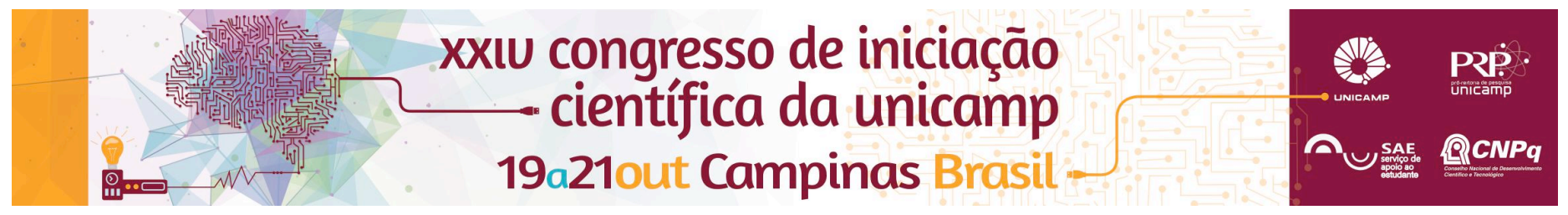

\title{
Development of a graphical user interface for neurofeedback training using near-infrared spectroscopy
}

\author{
Giovanni H. Scavariello*, João O. F. Pigatto, Mariana S. Pavan, Mônica A. Cotta, Gabriela Castellano, Rickson C. \\ Mesquita
}

\begin{abstract}
Neurofeedback (NFB) protocols are training protocols based on the user's own brain signals. More recently, NFB has been the object of many recent studies in neuroscience and has generated a lot of discussions about its effectiveness. The aim of this work was to develop a graphical user interface for NFB training using real-time near-infrared spectroscopy (NIRS).
\end{abstract}

\section{Key words:}

Neurofeedback, near-infrared spectroscopy, neuromodulation

\section{Introduction}

Neurofeedback (NFB) is a specific technique within the biofeedback protocols, which makes use of the ability of an individual to train a specific set of skills using information received from his/her own practice, for example, how a ballerina trains her poses in front of a mirror. NFB is a form of biofeedback in the sense that an individual can voluntarily control his/her own brain activation. This allows brain function training, aiming to reduce disease symptoms, such as those from epilepsy ${ }^{[1]}$ and ADHD ${ }^{[2]}$ or to enhance performance, such as training concentration and anxiety control. In NFB, the subject is presented with a measure of brain activity and tries to change this signal towards a desired goal (increase or decrease). Typically, NFB protocols use electroencephalography (EEG) for obtaining brain signals and modulating activity training. In this study, we quantified brain activity with near-infrared spectroscopy (NIRS), which is a non-invasive spectroscopy technique able to extract hemodynamic information using nearinfrared light sources. The NIRS technique is based in the fact that in the near-infrared range of the electromagnetic spectrum, there is an absorption "window" in biological tissue (around 700 to $900 \mathrm{~nm}$ ), and the main chromophores are the oxygenated and deoxygenated versions of hemoglobin (oxyhemoglobin and deoxyhemoglobin respectively). When a brain region "activates", there is an increase in blood flow to this region, which increases the relative concentration of oxyhemoglobin in the region. Concentration changes of oxy and deoxyhemoglobin can therefore be tracked with NIRS, giving an indirect measure of how the brain is working. The main goal of this work was to develop and validate a user-friendly interface for real-time NFB using NIRS for research purposes.

\section{Results and Discussion}

Our result is a graphical user interface able to display any variable of interest obtained through NIRS in real time. The interface was developed from an open NIRS data acquisition code for the TechEn CW6 System, using MATLAB. This interface already plotted the hemodynamic response in real time through variations in light intensity and showed the probe geometry in use. we added an extra figure in the software which plots the feedback variable in real time. The feedback is displaved in the form of a multi bar graph, in order to provide the subject with a sense of progression throughout the session. Figure 1 shows the developed interface.

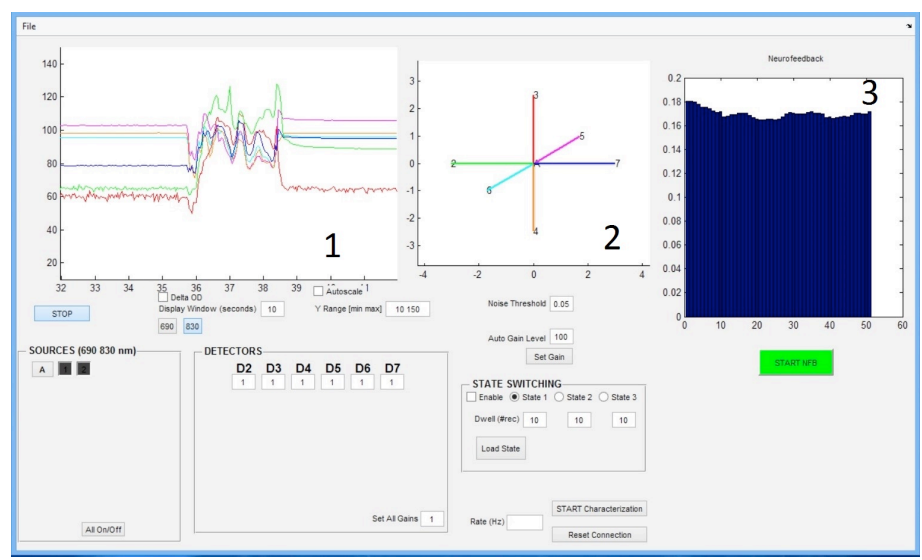

Figure 1. Illustration of the developed interface plotting 1) Optical intensity, 2) The probe geometry and 3) A feedback dummy variable.

\section{Conclusions}

In this work, we developed an interface for NFB training using NIRS. Up to now, the interface is up and running. The interface presents the NIRS signal in real-time via computer to a subject. The next steps will be to recruit volunteers for NFB training sessions, and to determine the NIRS parameter that best represents brain activity for NFB purposes.

\section{Acknowledgement}

Acknowledgements to CEPID BRAINN, Neurophysics group, LOB, FAPESP, CAPES and CNPq.

\footnotetext{
Kotchoubey, B., Schneider, D., Schleichert, H., Strehl, U., Uhlmann, C. Blankenhorn, V., Fröscher, W., Birbaumer, N. Epilepsy Res. 1996, 25(3): 269276.

Lansbergen, M.M., van Dongen-Boomsma, M., Buitelaar, J.K., SlaatsWillemse, D. J. Neural Transm. 2011, 118(2):275-284.
} 